\title{
Next Generation Superconductors for High Field Applications
}

R. Flükiger and B. Seeber of the Department of Condensed Matter Physics and the Institute of Applied Physics, University of Geneva, describe the status of oxide and sulphide superconducting tapes and wires.

The economic generation of steady state magnetic fields by means of superconductors is presently limited to about $20 \mathrm{~T}$. The main reason for this restriction is related to the intrinsic upper critical field $\left(\mathrm{B}_{\mathrm{c} 2}\right)$ of today's commercially available superconductors based on $\mathrm{Nb}_{3} \mathrm{Sn}$ (see Fig. 1). For higher fields at $4.2 \mathrm{~K}$, a new generation of superconducting materials must be developed, prime candidates being $\mathrm{PbMo}_{6} \mathrm{~S}_{8}$ (PMS) crystallizing as the Chevrel phase and high temperature superconducting (HTSC) copper oxide compounds with a layered structure. The former has a $B_{\mathrm{c} 2}$ at $4.2 \mathrm{~K}$ above $50 \mathrm{~T}$ which is nearly isotropic owing to the rhombohedral crystal structure with a rhombohedral angle close to $90^{\circ}$. The two-dimensional character of HTSC's implies a very strong anisotropy: for example, the compound $\mathrm{YBa}_{2} \mathrm{Cu}_{3} \mathrm{O}_{7}$ has a $B_{\mathrm{c} 2}$ at 4.2 $K$ reaching $100 \mathrm{~T}$ perpendicular to the $c$-axis, and 25-60 T in the parallel direction.

Another important parameter for applications is the transport critical current density $J_{c}$ at the required magnetic field. While supporting the generated magnetic field, the superconductor used to wind a magnetic solenoid must be in the form of a long wire with a reasonably high and constant $J_{c}$ (typically $5 \times 10^{8} \mathrm{~A} / \mathrm{m}^{2}$ ) over several hundred metres. Obtaining such characteristics at $77 \mathrm{~K}$ using liquid nitrogen as the cryogen instead of at $4.2 \mathrm{~K}$ with liquid helium presents obvious economic advantages. However, the strong temperature dependence of $J_{c}$ and $B_{c 2}$ implies that for many high field applications, the main operation range will be limited to $4.2 \mathrm{~K}$ for the foreseeable future. Nonetheless, achieving more modest fields (a few tesla) at $77 \mathrm{~K}$ offers important opportunities.

We review here briefly the status of the development of HTSC tapes and PMS wires for high field applications at $4.2 \mathrm{~K}$. In the case of HTSC's, one is usually considering highly textured, monofilamentary or multifilamentary layers. For the Chevrel compounds, a wire of circular cross-section can be employed.

\section{HTSC Tapes}

From reported data on HTSC's, three candidates have been retained with applications at $4.2 \mathrm{~K}$ and $77 \mathrm{~K}$ in mind, namely $(\mathrm{Bi}, \mathrm{Pb})_{2} \mathrm{Sr}_{2} \mathrm{Ca}_{2} \mathrm{Cu}_{3} \mathrm{O}_{\mathrm{x}}$ - so-called $\mathrm{Bi}(2223)$, the $\mathrm{TI}$ substituted version - so-called $\mathrm{TI}(2223)$, and $\mathrm{YBa}_{2} \mathrm{Cu}_{3} \mathrm{O}_{7}$.

Melt-textured samples of $\mathrm{YBa}_{2} \mathrm{Cu}_{3} \mathrm{O}_{7}$ prepared by adjusting the solidification conditions of the molten compound to create the appropriate crystallographic anisotropy and crystal structure exhibit fairly high $J_{c}$ 's, e.g., $3 \times 10^{8} \mathrm{~A} / \mathrm{m}^{2}$ at $77 \mathrm{~K}$ under an external field of $1 \mathrm{~T}$ [1]. Unfortunately, the poor mechanical properties of this compound render its deformation very difficult. This explains why, in contrast to melt textured samples, silver-sheathed tapes have so far exhibited disappointingly low $J_{c}$ 's owing to very low degrees of crystal texturing and to the presence of superconducting weak links at grain boundaries.

The situation is different for the two (2223) superconductors which are mechanically softer than the $\mathrm{Y}$-based oxide and can be easily brought into a textured configuration by choosing the correct sequence of pressing and annealing steps for the powder-in-tube preparation technique, where a metallic can containing the HTCS powder is thermo-mechanically processed. The mechanical and physical properties of both (2223) phases are very similar, but a substantial difference resides in the formation mechanism. The TI based phase can be formed by a melting process while $\mathrm{Bi}(2223)$ requires a reaction of the solid $\mathrm{Bi}(2223)$ phase with a liquid phase mixture and is stabilized by the addition of $\mathrm{Pb}$ [2]. It is known that $\mathrm{Ag}$ lowers the melting point of $\mathrm{Bi}(2223)$ but for the formation of this phase in $\mathrm{Ag}$ sheathed ribbons, the important feature is the lowering of the melting point of the liquid phases, thus promoting $\mathrm{Bi}(2223)$ grain growth. In spite of the more complex formation conditions in $\mathrm{Bi}(2223)$ with respect to $\mathrm{TI}(2223)$, most publications are restricted to the former as the toxicity of $\mathrm{TI}$ calls for much higher safety standards.

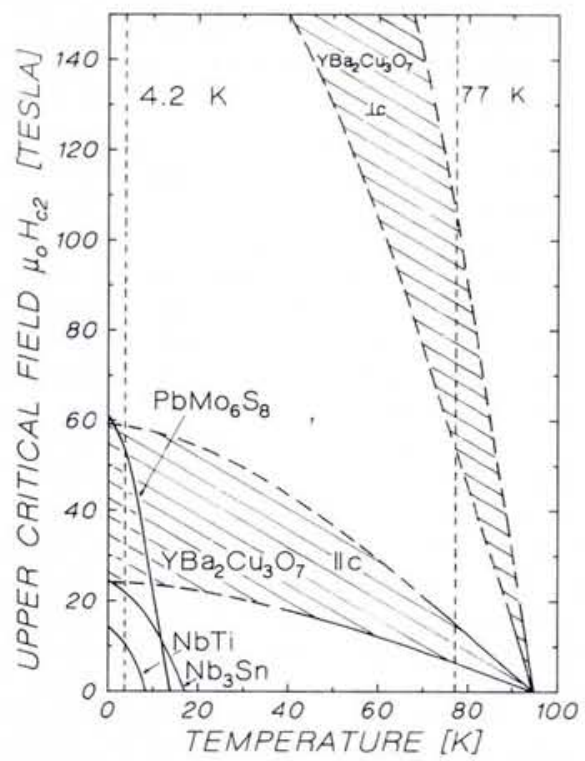

Fig. 1 - Upper critical field $B_{c 2}$ as a function of temperature for the commercial superconductors $\mathrm{NbTi}$ and $\mathrm{Nb}_{3} \mathrm{Sn}$. Data for the $\mathrm{PbMo}_{6} \mathrm{~S}_{8}$ Chevrel phase are also compared with those for the high temperature oxide superconductor $\mathrm{YBa}_{2} \mathrm{Cu}_{3} \mathrm{O}_{7}$ with the magnetic field both parallel and perpendicular to the the $c$-axis. The hatched areas correspond to uncertainties related to the criterion used to define $B_{c 2}$ and to the extrapolation scheme.

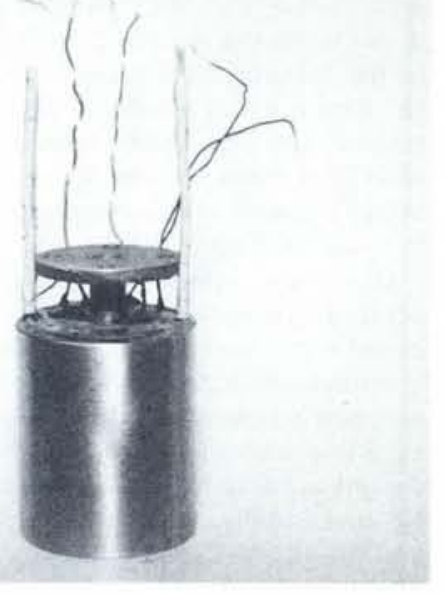

Fig. 2 - An $80 \mathrm{~mm}$ diameter coil made from a HTSC wire. It generated 23.225 T at $4.2 \mathrm{~K}$ with a $23.0 \mathrm{~T}$ backup field meaning that, with sufficient material, a $23.225 T$ magnet could be built (courtesy of Sumitomo Electric Industries, Osaka, Japan).

Relatively high $J_{c}$ values have been obtained at $77 \mathrm{~K}$ for $\mathrm{Tl}$ and $\mathrm{Bi}(2223)$ tapes produced by a special type of powder-in-can process, namely the press-and-anneal method. Powders, which may comprise mainly the (2223) phase or a blend of $\mathrm{CuO}$ and some other oxides, are packed into a $\mathrm{Ag}$ tube which is swaged, drawn and rolled to roughly $0.1 \mathrm{~mm}$ thick tape. The tapes are then subjected to one or more pressing/heat treatment cycles. For Ag-sheathed TI(2223) tapes, $J_{c}(77 \mathrm{~K}, 0 \mathrm{~T})$ values of $1.4 \times 10^{8}$ $A / \mathrm{m}^{2}$ have been reported [3] while even higher values were found for $\mathrm{Pb}$-stabilized $\mathrm{Bi}(2223)$ tapes $\left(4.7 \times 10^{8} \mathrm{~A} / \mathrm{m}^{2}[4], 3.3 \times\right.$ $10^{8} \mathrm{~A} / \mathrm{m}^{2}$ [5]); values up to $4.0 \times 10^{8} \mathrm{~A} / \mathrm{m}^{2}$ have been achieved in our laboratory. It must be noted, however, that these high values of $J$ have so far only been found for tapes less that $40 \mathrm{~mm}$ in length where pressures as large as $50 \mathrm{kbar}$ can be applied.

Attempts to reach these values by rolling steps alone (without intermediate pressing) have been unsuccessful. $J_{c}(77 \mathrm{~K}, 0 \mathrm{~T})$ values for tapes longer than the $10 \mathrm{~m}$ required to wind small laboratory magnets have todate reached on the order of $1 \times 10^{8} \mathrm{~A} / \mathrm{m}^{2}$. Nevertheless, small superconducting magnets producing $0.2 \mathrm{~T}$ at $4.2 \mathrm{~K}$ have already been produced in Japan (Fig. 2).

The variation of $J_{C}$ with the applied magnetic field $B$ perpendicular to the $c$-axis (as is the situation in a wound magnet solenoid) is indicated in Fig. 3 for $\mathrm{Bi}(2223)$ tapes: there is a fairly strong dependence up to about $1 \mathrm{~T}$. However, $J_{c}$ is strongly dependent on the orientation of the magnetic field (Fig. 4). It follows that the limiting factor for the production of fields above $0.2 \mathrm{~T}$ at $77 \mathrm{~K}$ is the occurrence of magnetic field components parallel to the $c$-axis which arise at both ends of a wound coil. Particularly elaborate configurations have to be developed to solve this problem. Nonetheless, considerable effort is being undertaken in order to further enhance $J_{c}$ values of HTSC's and to improve the mechanical properties of $\mathrm{Ag}$ sheathed $\mathrm{Bi}(2223)$ tapes with applications both at $77 \mathrm{~K}$ and $4.2 \mathrm{~K}$ in view. 


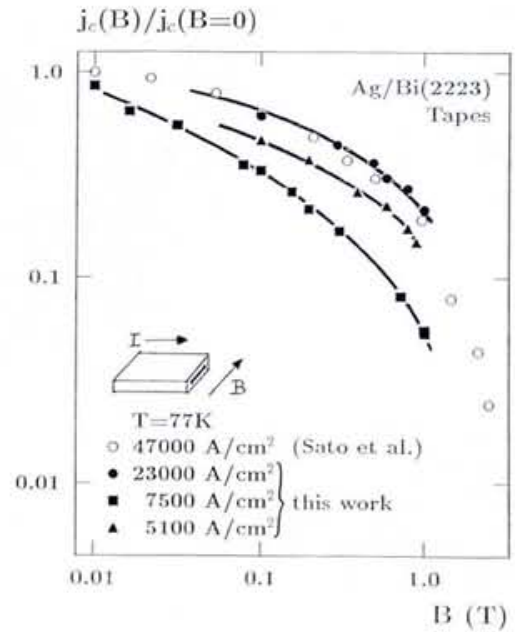

Fig. 3-Normalized critical current density $J_{c}$ at a temperature $T=77 \mathrm{~K}$ versus the applied magnetic field $B$ for $A g$-sheathed $B i$ (2223) high temperature oxide superconductor tapes with $B$ perpendicular to the c-axis [7]. Densities at zero field are given at the lower left-hand side.

$I_{c}(\Theta) / I_{c}\left(\Theta=90^{\circ}\right)$

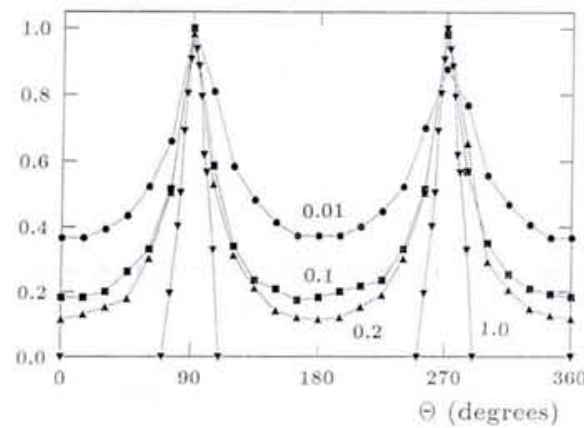

Fig. 4-Normalized critical current density as a function of the orientation $\theta$ of the magnetic field for a Ag sheathed Bi(2223) tape [7]. An angle $\theta=90^{\circ}$ corresponds to a field $B$ perpendicular to the $c$-axis.

\section{PMS Wires}

Chevrel phase superconductors have been under intensive investigation over the the past 10 years or so. A multinational EUREKA project was launched in 1986 to develop an industrial-scale production capability for PMS-based superconducting wire capable of generating fields above $20 \mathrm{~T}$. Monofilamentary wires of up to one kilometre in length have been produced and coils wound from the conductor demonstrated. The best results reported to-date by the EUREKA project team are for $\mathrm{Cu} / \mathrm{Nb}$ sheathed material produced using the conventional powder-in-tube method [8]. Other matrix materials such as $\mathrm{Mo} /$ stainless steel and $\mathrm{Ta} / \mathrm{Cu} /$ stainless steel sheaths gave similar results $[9,10]$. The $\mathrm{Mo}, \mathrm{Nb}$ or Ta acts as a diffusion barrier while the steel ensures matching of the thermal expansion between the core and the sheath to suppress thermally induced stresses on cooling from heat treatment temperatures: copper acts as a thermal stabiliser. Starting powders are either a blend of metals and sulphide compounds or pre-reacted PMS, and heat treatments after wire fabrication by swaging, extrusion and drawing are needed to react the powders or to recover superconducting properties.

Critical current densities at $4.2 \mathrm{~K}$ exceeding those for $\mathrm{Nb}_{3} \mathrm{Sn}$ superconductors have been achieved for short lengths $(25 \mathrm{~m})$ of $\mathrm{Nb} / \mathrm{Cu}$ sheathed monofilamentary PMS material at $20 \mathrm{~T}$ (Fig. 5). Important advantages with respect to $\mathrm{Nb}_{3} \mathrm{Sn}$ include almost no field dependence of $J$ under uniaxial strain in the 8-24 Trange (see Fig. 6) and a $30 \%$ higher intrinsic elastic limit. Calculations have also shown that the superconducting properties in monofilamentary PMS wire with today's usual filament diameters are intrinsically stable above $20 \mathrm{~T}$ at $4.2 \mathrm{~K}$.

D. Cattani [12] using flux profile measurements has demonstrated that $J_{c}$ within PMS grains in a bulk sample is about ten times larger than $J_{c}$ for the best wires, and that it increases with an increase in $B_{\mathrm{c} 2}^{*}$, the upper critical field derived from $A C$ susceptibility measurements. Depending on the preparation, $B_{\mathrm{c} 2}^{*}$ is also significantly smaller than the virtually sample independent $B_{\mathrm{c} 2}$ values obtained using calorimetry. It is believed that the low $B_{c 2}^{*}$ value reflects the upper critical field of the superconductor at the grain boundaries so, at present, the most important obstacle in improving $J_{c}$ appears to reside in the local properties at grain boundaries. The coherence length of PMS is approximately the same as in the ab-plane of HTSC's (and much larger than for the $c$-direction) so the problems of grain boundary limited $J_{c}$ 's in the two materials show some similarity. Extensive work is now in progress to investigate the grain boundary aspects.

\section{Conclusions}

Based on available $B_{c 2}$ and $J_{c}$ data (Figs 1 and 5) one can conclude that both classes of superconductors, HTSC and Chevrel phases, could be used to produce fields above $20 \mathrm{~T}$ at $4.2 \mathrm{~K}$. From the present results, the advantage of HTSC materials is the weaker decrease of $J_{c}$ above $20 \mathrm{~T}$ and

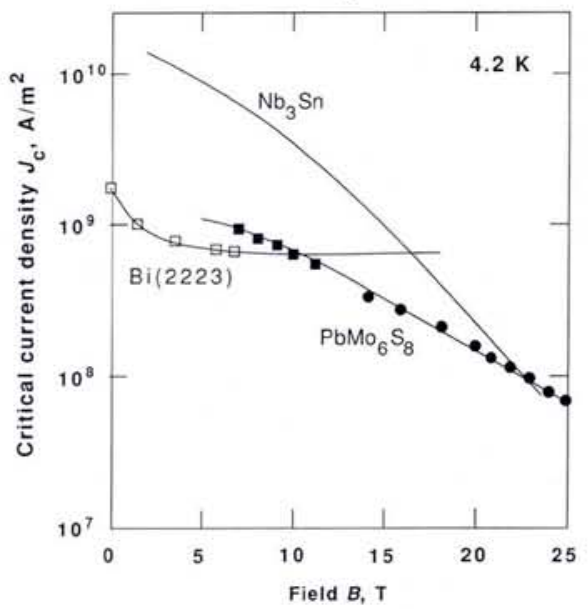

Fig. 5 - Comparison of the critical current density $J_{c}$ at $4.2 \mathrm{~K}$ as a function of the magnetic field for a $\mathrm{Nb} / \mathrm{Cu}$ sheathed monofilamentary $\mathrm{PbMo}_{5} \mathrm{~S}_{8}$ Chevrel phase wire $[8,10]$, a multifilamentary $\mathrm{Nb}_{3} \mathrm{Sn}$ wire and Ag-sheathed Bi(2223) tape [7]. $J_{c}$ was determined for the superconducting crosssection.

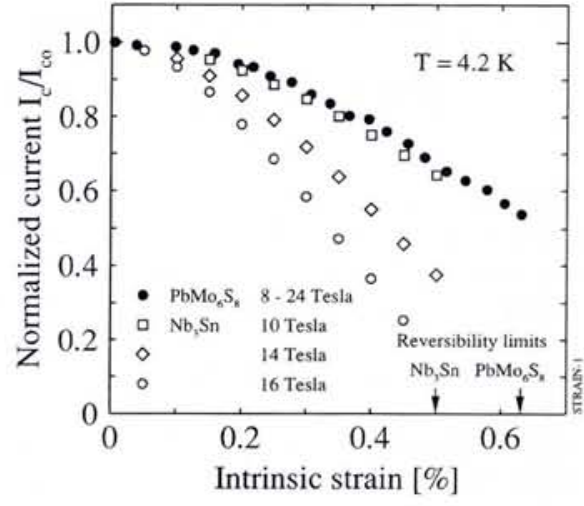

Fig. 6 - Critical current $l_{c}$ normalized with respect to $I_{c o}$, the critical current at zero strain, versus intrinsic strain at $4.2 \mathrm{~K}$ for monofilamentary $\mathrm{Ta} / \mathrm{Cu} /$ stainless steel sheathed $\mathrm{PbMo}_{6} \mathrm{~S}_{8}$ Chevrel phase and $\mathrm{Nb}_{3} \mathrm{Sn}$ multifilamentary superconducting wires. The reversibility limits may be shifted to higher or lower values depending on the thermally induced pre-stress [10, 13].

the larger temperature difference between the critical temperature $T_{c}$ and the operation temperature. However, the isotropic properties of PMS allow the use of round wires which presents some advantages for industrial fabrication and possibly for particular applications. From the data obtained on small test coils $[8,12]$, and the agreement between $J_{c}$ in these coils and in short samples, it follows that a high degree of homogeneity can now be obtained in PMS wires, while long $\mathrm{Bi}(2223)$ tapes with high $J_{c}$ still show a considerable variation with the length.

The development of HTSC tapes is presently directed towards high current leads at $77 \mathrm{~K}$ (e.g. between liquid $\mathrm{N}_{2}$ and $\mathrm{He}$ ) and towards solenoids with moderate magnetic fields, the medium term goal being to achieve field values comparable to those produced by conventional superconducting magnets.

\section{REFERENCES}

[1] Murakami M. et al., IEEE Trans. Magn. MAG-27 (1991) 1479.

[2] Sunshine S.A. et al., Phys. Rev. B 38 (1988) 893.

[3] Okada M., Jpn. J. Appl. Phys. 29 (1990) 2732.

[4] Sato K. et al., IEEE Tras. Magn. MAG-27 (1991) 1231.

[5] Heine K. et al., Adv. Cryo. Engng. 38 (1991), to be published.

[6] Yamada Y., Obst B. and Flükiger R., Superconductor Sci. \& Tech. 4 (1991) 165. [7] Flükiger R. et al., VIth. Int. Workshop on Critical Currents, Cambridge, UK (1991); to be published in Superconductor Sci. \& Tech. [8] Genevey P., Vallier J.C. et al. (private communication).

[9] Seeber B. et al., Proc. MRS Meeting on Advanced Materials 6 (1989) 83.

[10] Rimikis G. et al., IEEE Trans. Magn. MAG-27 (1991) 1116.

[11] Ekin J.W., Yamashita T. and Hamasaki K., IEEE Trans. Magn. MAG-21 (1985) 474. [12] Cattani D. et al., IEEE Trans. Magn. MAG-27 (1991) 950.

[13] Goldacker W. et al., Proc. ICMAS (1991). 